\title{
Indicadores de Potencial de Aprendizagem Obtidos através da Avaliação Assistida
}

\author{
Silvia Helena Tortul Ferriolli ${ }^{12}$ \\ Maria Beatriz Martins Linhares \\ Sonia Regina Loureiro \\ Edna Maria Marturano \\ Universidade de São Paulo, Ribeirão Preto
}

\begin{abstract}
Resumo
O objetivo deste trabalho foi detectar indicadores de potencial cognitivo de crianças com queixa de dificuldade de aprendizagem escolar, utilizando procedimento combinado de avaliação psicométrica (Raven) e avaliação assistida (Jogo de Perguntas de Busca com Figuras Diversas - PBFD). Foram avaliadas 20 crianças de oito a 11 anos, encaminhadas para atendimento psicológico na área da Saúde com queixa de dificuldade de aprendizagem. O PBFD foi delineado em fases e realizado antes (avaliação) e após (reavaliação) uma intervenção psicopedagógica de curta duração. Houve variações de desempenho na avaliação psicométrica, com tendência à classificação "definidamente abaixo da média". Na avaliação assistida, nos momentos da avaliação e da reavaliação, houve variações nos perfis de desempenho cognitivo, indicando diferenças individuais quanto às dificuldades e recursos cognitivos. Discriminou-se um subgrupo de crianças que precisaram de pouca ajuda e monitoramento, daquele de crianças que precisaram de mais assistência para implementar estratégias eficientes na resolução de problemas. Palavras-chave: Avaliação cognitiva; avaliação assistida; dificuldade de aprendizagem.
\end{abstract}

\section{Indicators of Learning Potential Obtained through Assisted Assessment}

\begin{abstract}
This research intended to detect indicators of cognitive potential of children with school learning disability complaints, employing combined procedures of psychometric assessment (Raven) and assisted assessment (Constraint-seeking questions game with several figures - PBFD). Twenty children with learning disability complaints, aged from eight to 11 years, were assessed, all referred for psychological evaluation in Health Services. PBFD was delineated by phases and carried out before (assessment) and after (re-assessment) a short duration psychopedagogic intervention. There was a variation in the performance of children in the psychometric assessment, with a tendency of predominance of the classification "definitely below the average". In the assisted assessment, there was variation in the cognitive performance profile, showing individual differences in cognitive difficulties and resources, both assessment and re-assessment moments. A subgroup of children needing few help and monitoring was distinguished, in contrast with a group that needed more assistance to implement efficient strategies for problem resolution.

Keywords: Cognitive assessment; assisted assessment; learning disability.
\end{abstract}

Tem sido constatada a existência de alta demanda para atendimento psicológico em serviços públicos de saúde, referente a crianças com queixa de dificuldade de aprendizagem escolar, na faixa etária de sete a doze anos (Graminha \& Martins, 1997; Linhares, Parreira, Maturano \& Sant'Anna, 1993; Lopez, 1983; Santos, 1990). O aumento da procura de atendimento para esses casos indica a necessidade de um aprofundamento no estudo da natureza e das características dos problemas

\footnotetext{
${ }^{1}$ Endereço para correspondência: Av. 9 de Julho, 980, Ribeirão Preto, SP, 14025-000. Fone: (16) 6250309. E-mail: linharesmbm@iname.com

${ }^{2} \mathrm{O}$ texto consiste em parte da Dissertação de Mestrado da primeira autora (bolsista da CAPES) sob a orientação da segunda, e do Projeto Integrado do CNPq (Processo no 5222343/95-3).
}

apresentados por essas crianças, para que se possa fundamentar procedimentos de prevenção e intervenção.

As crianças encaminhadas para atendimento psicológico na área da Saúde, com queixa de dificuldade de aprendizagem, constituem um grupo heterogêneo com variações quanto as suas reais dificuldades e capacidades cognitivas (Marturano, Loureiro, Linhares \& Machado, 1997). Sendo assim, a avaliação psicológica a que são submetidas inclui a avaliação de aspectos cognitivos, com o objetivo de identificar a possível existência de dificuldades na área intelectual. Os testes psicométricos, que geralmente são empregados no estudo desses casos, oferecem uma compreensão parcial do funcionamento cognitivo, devido à natureza genérica das informações que são por eles fornecidas. Esses se 
concentram em habilidades e conhecimentos acumulados pela criança até o momento da avaliação. Em função disso, torna-se cada vez mais recomendado e necessário o uso de procedimentos de avaliação cognitiva que contribuam não apenas para identificar dificuldades, mas também dimensionar recursos potenciais do seu funcionamento cognitivo. Para esse dimensionamento tem sido propostos procedimentos que permitam a análise de estratégias de resolução de problemas e que apreendam a sensibilidade da criança à instrução (Campione, 1989; Lunt 1994), no intuito de contribuir com informações que ajudem a traçar diretrizes de mediação de aprendizagem adequadas às necessidades individuais da criança.

$\mathrm{Na}$ área de avaliação cognitiva, portanto, tem havido, recentemente, um interesse crescente em procedimentos processuais, dinâmicos e interativos, destacando-se entre esses a avaliação assistida. Esta modalidade de avaliação fundamenta-se nos conceitos de aprendizagem mediada e zona de desenvolvimento proximal de Vygotsky (1978/ 1988). O examinador, utilizando um conjunto de estratégias instrucionais, temporárias e ajustáveis ao desempenho da criança, ajuda a revelar o seu desempenho potencial, fazendo-a alcançar um grau crescente de autonomia em situações de resolução de problemas (Brown \& Campione, 1986; Campione, 1989; Linhares, 1996). Dessa forma, é possível aumentar a compreensão sobre as estratégias cognitivas utilizadas pela criança durante a realização de solução de tarefas, complementando informações que foram obtidas através de avaliação psicométrica tradicional, com informações a respeito da sensibilidade à instrução e de indicadores do potencial cognitivo dos examinandos. Esses indicadores referem-se à quantidade e tipo de ajuda necessária para que a criança solucione efetivamente determinada tarefa, à relevância das estratégias cognitivas utilizadas na sua execução e os tipos de tentativas de solução realizadas (Linhares, 1996). Através da avaliação assistida, pode-se identificar variações individuais e intragrupo em crianças com desvantagens, tais como deficiência mental, dificuldade de aprendizagem ou desvantagem cultural (Brown \& Ferrara, 1985; Santa Maria \& Linhares, 1999; Swanson, 1995).

Entre as tarefas que permitem a identificação dessas variações e de indicadores de potencial de aprendizagem, destacam-se, entre outras, aquelas que envolvem perguntas de busca de informação e restrição de alternativas. Os jogos de perguntas de busca têm demonstrado ser um procedimento sensível para revelar indicadores de potencial para a aprendizagem em crianças com dificuldade de aprendizagem em situação de avaliação assistida (Barton, 1988; Courage, 1989; Gera \& Linhares, 1998; Linhares, 1996; Linhares, Santa Maria, Escolano \& Gera, 1998; Santa Maria \& Linhares, 1999).

Estudos têm demonstrado que em situação de avaliação, ao receber ajuda instrucional temporária e ajustável às suas necessidades individuais, há crianças que conseguem melhorar o seu desempenho, enquanto outras necessitam de assistência mais intensiva e prolongada (Escolano, 2000; Feuerstein, Rand, Hoffman \& Miller, 1979; Linhares 1998a).

Segundo Feurstein, Rand, Hoffman e Miller (1980), as experiências vividas durante o processo de mediação permitem à criança modificar as suas estruturas cognitivas, e, conseqüentemente, adaptar-se a novos modos de funcionamento. A plasticidade cognitiva varia em grau de uma criança para outra, e está diretamente relacionada à capacidade individual de se beneficiar da ajuda recebida durante o processo de mediação.

A intervenção psicopedagógica dirigida à criança com dificuldade de aprendizagem visa promover ajuda continuada à criança, na medida em que representa uma situação protegida de ensino-aprendizagem, com objetivo de dessensibilizar a criança, diminuindo a ansiedade frente à tarefa de aprender e propiciar o desenvolvimento de habilidades e transmitir conhecimentos (Linhares, 1998b). Dessa forma, a intervenção psicopedagógica combinada com a avaliação assistida pode ampliar a possibilidade de detectar recursos potenciais cognitivos da criança, muitas vezes encobertos por situações aversivas de ensino experimentadas anteriormente.

Considerando-se o exposto, o presente trabalho teve por objetivo identificar indicadores de potencial cognitivo em um grupo de crianças encaminhadas para atendimento psicológico apresentando queixa de dificuldade de aprendizagem escolar, através de procedimento combinado de avaliação, utilizando teste psicométrico de inteligência e situação estruturada de avaliação cognitiva assistida, sendo esta última realizada antes e após um período de intervenção psicopedagógica de curta duração.

\section{Método}

\section{Participantes}

Foram avaliadas 20 crianças (12 meninos e 8 meninas), de oito a 11 anos ( $M d=8$ anos e 10 meses), alunas de primeira à quarta série $\left(M d=3^{\mathrm{a}}\right.$ série $)$ do ensino fundamental de escola pública, com queixa de dificuldade de aprendizagem escolar, encaminhadas para atendimento psicológico junto a um Ambulatório de Psicologia Infantil do Hospital das Clínicas da FMRP. As crianças não 
apresentavam problemas neurológicos, genéticos ou psiquiátricos e não estavam recebendo atendimento especializado para dificuldades de aprendizagem em outro serviço.

\section{Material}

Foram utilizados os seguintes materiais: Matrizes Progressivas Coloridas de Raven-Escala Especial (Angelini, Alves, Custódio \& Duarte, 1987); Jogo de Perguntas de Busca com Figuras Diversas (PBFD) (Gera \& Linhares, 1998); gravador e fitas cassetes. A descrição do Jogo de Perguntas de Busca com Figuras Diversas encontra-se no Anexo A.

\section{Procedimento}

O esquema de coleta de dados incluiu duas etapas distintas. Na primeira etapa do procedimento, foram realizadas duas sessões de avaliação cognitiva: na primeira sessão, aplicou-se o Raven segundo as normas de padronização brasileira. Na segunda sessão, que ao longo deste trabalho será denominada avaliação, foi aplicado o procedimento de avaliação cognitiva assistida em situação de resolução de problemas, utilizando-se o Jogo de Perguntas de Busca com Figuras Diversas (Gera \& Linhares, 1998).

A segunda etapa do procedimento de avaliação cognitiva (denominada reavaliação), ocorreu após uma intervenção psicopedagógica de curta duração ${ }^{3}$, quando as crianças foram reavaliadas através do mesmo procedimento de avaliação assistida utilizado na primeira etapa (avaliação).

O procedimento de avaliação assistida em situação de resolução de problemas foi delineado segundo o método estruturado (Campione \& Brown, 1990; Linhares, 1998c; Tzuriel \& Klein, 1987), incluindo as seguintes fases durante a avaliação: Inicial sem Ajuda (SAJ), Assistência (ASS) e Manutenção (MAN). Na fase inicial sem ajuda, foi avaliado o desempenho real da criança, uma vez que esta trabalhou sozinha de forma independente, segundo instruções padronizadas.

A fase de assistência, por sua vez, tem por objetivo avaliar indicadores de desempenho potencial da criança, quando ela está trabalhando em conjunto com a examinadora. Nesta fase, era oferecido pela examinadora um suporte instrucional adicional, temporário e ajustável

\footnotetext{
${ }^{3} \mathrm{O}$ trabalho de intervenção psicopedagógica foi realizado no Ambulatório de Psicologia Infantil do HCFMRP, com grupos de no máximo quatro crianças, que eram atendidos uma vez por semana, de acordo com a "perspectiva desenvolvimentista"de intervenção psicopedagógica proposta por Linhares (1998b). O número total de atendimentos variou de 11 a 15 sessões, com duração de noventa minutos cada uma.
}

ao desempenho da criança, com o objetivo de melhorar as condições de avaliação e, conseqüentemente, favorecer a revelação de indicadores de desempenho potencial e de autonomia em situações de resolução de problemas. A mediação da aprendizagem era realizada através de cinco níveis de ajuda crescentes, regulados de acordo com a necessidade da criança. Os cinco níveis de ajuda, em ordem crescente de assistência da examinadora eram os seguintes: feedback informativo, análise comparativa, exemplo de pergunta relevante, retirada dos cartões e demonstração de um modelo de pergunta (Santa Maria \& Linhares, 1999). O avanço para um nível superior de mediação não excluía a possibilidade de utilização de níveis anteriores, uma vez que esses podem ser cumulativos. As definições e exemplos dos níveis de ajuda encontram-se no Anexo B.

A fase de manutenção tem por objetivo avaliar o nível de desempenho da criança quanto à manutenção da aprendizagem das estratégias de perguntas de busca de informação. Nesta fase, a ajuda era suspensa e a criança devia novamente resolver a tarefa sozinha, segundo as instruções padronizadas, sem intervenções adicionais da examinadora.

As sessões de avaliação assistida foram gravadas e as verbalizações da criança e da examinadora foram posteriormente transcritas para análise do desempenho da criança na tarefa.

\section{Análise dos Dados}

O desempenho no Raven foi avaliado de acordo com as normas de padronização brasileira, em termos de percentil.

Os indicadores de desempenho na tarefa de resolução de problemas (PBFD), tanto na avaliação quanto na reavaliação, foram quantificados em termos de taxa ou proporção, quanto a: número de perguntas de busca formuladas, relevância das estratégias de perguntar por sua eficiência ou poder de restrição de alternativas e tipos de tentativas de solução (Linhares, 1996; Santa Maria \& Linhares, 1999). Com base nesses dados, foi realizada uma análise comparativa das estratégias de solução de cada sujeito, apresentadas na fase $\mathrm{SAJ}$ em relação às fases ASS e MAN, respectivamente. A significância das comparações entre as diferentes fases foi verificada através da prova não paramétrica de Wilcoxon para amostras pareadas $(p \leq 0,05)$. Foram identificados, tanto na avaliação quanto na reavaliação, perfis de desempenho cognitivo potencial individual de cada criança, podendo ser: a) alto-escore, crianças que apresentavam bom desempenho logo na fase inicial sem ajuda, e que mantinham o bom desempenho na fase de manutenção; 
b) ganhador mantenedor, crianças que demonstravam melhora no desempenho cognitivo na fase de assistência e mantinham essa melhora na fase de manutenção; c) ganhador dependente da assistência, crianças que melhoravam seu desempenho com a ajuda da examinadora mas não mantinham a melhora após a suspensão da ajuda na fase de manutenção e d) não ganhador, crianças que não melhoravam ou apresentavam pouca melhora no seu desempenho, não a mantendo após a suspensão da ajuda da examinadora (Santa Maria \& Linhares, 1999). Esses perfis foram definidos operacionalmente por Escolano (2000).

Os perfis de desempenho cognitivo de cada criança obtidos na avaliação foram comparados com os obtidos na reavaliação através do teste não paramétrico de Wilcoxon.

A prova do Coeficiente de Correlação de Postos de Spearman permitiu analisar a correlação entre os perfis de desempenho cognitivo na avaliação e na reavaliação, respectivamente, e as seguintes variáveis: percentis no Raven e idade cronológica das crianças.

\section{Resultados}

A Tabela 1 reúne os indicadores de desempenho das crianças na resolução de problemas do PBFD nas diferentes fases (SAJ, ASS e MAN) e as comparações entre elas, na avaliação.

Na Tabela 1, observa-se que na fase SAJ, as crianças realizaram quatro perguntas em média por arranjo, mais perguntas relevantes do que não relevantes (repetidas, irrelevantes e incorretas) e predominantemente tentativas incorretas.

Comparando-se a fase SAJ com a fase ASS, verificase nesta última um aumento significativo das proporções medianas de perguntas relevantes e de tentativas corretas de solução e a conseqüente redução de tentativas incorretas e corretas ao acaso. A proporção de perguntas incorretas apresentou aumento significativo da fase SAJ para a ASS; apesar deste aumento, as perguntas incorretas se mantiveram em proporção inferior à das perguntas relevantes.

$\mathrm{Na}$ comparação entre as fases SAJ e MAN, por sua vez, nota-se a mesma tendência. $\mathrm{Na}$ fase MAN (após a suspensão da ajuda da examinadora), houve um aumento significativo na proporção de perguntas relevantes e de tentativas corretas e, em contrapartida, uma redução significativa de tentativas incorretas.

A Tabela 2 mostra os indicadores de desempenho das crianças na resolução do PBFD nas diferentes fases (SAJ, ASS e MAN) e as comparações entre elas, na reavaliação.

De acordo com a Tabela 2, na fase SAJ as crianças realizaram quatro perguntas em média por arranjo, formularam mais questões relevantes do que não relevantes (repetidas, irrelevantes e incorretas), com predomínio das tentativas incorretas de solução. Quando se compara a fase ASS em relação à SAJ, verifica-se um aumento significativo das proporções mediana de

Tabela 1. Indicadores de Desempenho no PBFD - Mediana (Md) e Comparações entre Fases, na Avaliação $(N=20)$

\begin{tabular}{|c|c|c|c|c|c|}
\hline \multirow[t]{3}{*}{ Indicadores de Desempenho PBFD } & \multicolumn{3}{|c|}{ Fases } & \multicolumn{2}{|c|}{ Comparações* $(p)$} \\
\hline & $\overline{\mathrm{SAJ}}$ & ASS & MAN & SAJ ASS & SAJ MAN \\
\hline & $\mathrm{Md}$ & $\mathrm{Md}$ & $\mathrm{Md}$ & & \\
\hline $\begin{array}{l}\text { Número médio de perguntas de } \\
\text { busca por arranjo de figuras }\end{array}$ & 4 & 4 & 5 & 0,29 & 0,56 \\
\hline $\begin{array}{l}\text { Proporção dos tipos de } \\
\text { pergunta de busca }\end{array}$ & & & & & \\
\hline Relevante & 0,46 & 0,78 & 0,64 & $<0,001^{*}$ & $0,009 *$ \\
\hline Irrelevante & 0,08 & 0,06 & 0,13 & 0,66 & 0,12 \\
\hline Incorreta & 0,09 & 0,12 & 0,13 & $0,02 *$ & 0,08 \\
\hline Repetida & 0 & 0 & $0,04 *$ & 0,26 & 0,70 \\
\hline
\end{tabular}

Proporção dos tipos de tentativas

$\begin{array}{lccccc}\text { Correta } & 0 & 0,70 & 0,29 & <0,001 * & 0,002^{*} \\ \text { Incorreta } & 0,73 & 0,30 & 0,42 & <0,001 * & 0,02^{*} \\ \text { Correta ao acaso } & 0,24 & 0 & 0,15 & <0,001 * & 0,12\end{array}$

* Prova de Wilcoxon $(p \leq 0,05) ;$ SAJ = fase inicial sem ajuda; ASS = fase de assistência; MAN = fase de manutenção 
Tabela 2. Indicadores de Desempenho no PBFD - Mediana (Md) e Comparações entre Fases, na Reavaliação $(N=20)$

\begin{tabular}{|c|c|c|c|c|c|}
\hline \multirow[t]{3}{*}{ Indicadores de Desempenho PBFD } & \multicolumn{3}{|c|}{ Fases } & \multicolumn{2}{|c|}{ Comparações* $(p)$} \\
\hline & $\overline{\text { SAJ }}$ & ASS & MAN & \multirow[t]{2}{*}{ SAJ ASS } & \multirow[t]{2}{*}{ SAJ MAN } \\
\hline & $\mathrm{Md}$ & Md & $\mathrm{Md}$ & & \\
\hline $\begin{array}{l}\text { Número médio de perguntas de } \\
\text { busca por arranjo de figuras }\end{array}$ & 4 & 4 & 4 & 0,82 & $0,04 *$ \\
\hline $\begin{array}{l}\text { Proporção dos tipos de } \\
\text { pergunta de busca }\end{array}$ & & & & & \\
\hline Relevante & 0,75 & 0,90 & 0,75 & $0,02 *$ & 0,11 \\
\hline Irrelevante & 0,03 & 0,03 & 0,10 & 0,60 & $0,005^{*}$ \\
\hline Incorreta & 0,14 & 0,06 & 0,10 & $0,05^{*}$ & 0,53 \\
\hline Repetida & 0 & 0 & 0 & 0,79 & 0,31 \\
\hline \multicolumn{6}{|l|}{ Proporção dos tipos de tentativas } \\
\hline Correta & 0,07 & 0,89 & 0,78 & $0,001 *$ & 0,06 \\
\hline Incorreta & 0,50 & 0,11 & 0,11 & $0,003 *$ & 0,16 \\
\hline Correta ao acaso & 0,29 & 0 & 0,12 & $0,001 *$ & $0,05^{*}$ \\
\hline
\end{tabular}

* Prova de Wilcoxon $(p \leq 0,05) ; \mathrm{SAJ}=$ fase inicial sem ajuda; ASS = fase de assistência; MAN = fase de manutenção

perguntas relevantes e de tentativas corretas de solução, com correspondente redução significativa das proporções de perguntas incorretas e das tentativas incorretas e corretas ao acaso.

Comparando-se as fases SAJ e MAN, observa-se nesta última um aumento significativo no número médio de perguntas de busca por arranjo, enquanto que a alta proporção mediana de perguntas relevantes permaneceu inalterada. Houve um aumento significativo na proporção mediana de perguntas irrelevantes, embora esta seja pequena em relação à proporção de perguntas relevantes. Verifica-se, ainda, uma tendência significativa de aumento da proporção mediana das tentativas corretas e uma diminuição significativa das tentativas corretas ao acaso.

Cabe salientar que, na avaliação, as proporções acima de 0,70 de perguntas relevantes e acertos só foram observadas na fase ASS, isto é, com a ajuda da examinadora presente. $\mathrm{Na}$ reavaliação, por sua vez as proporções de perguntas relevantes situaram-se acima de 0,75 nas três fases (SAJ, ASS e MAN), respectivamente, e as proporções de acertos foram expressivamente altas (acima de 0,70) nas fases ASS e MAN.

A Figura 1 apresenta os perfis de desempenho cognitivo das crianças na tarefa PBFD, durante a avaliação e reavaliação, segundo a classificação em: alto-escore, ganhador mantenedor, ganhador dependente da assistência e não ganhador.

Observa-se na Figura 1 que, na avaliação, cerca de 0,25 das crianças foram classificadas como ganhador mantenedor,

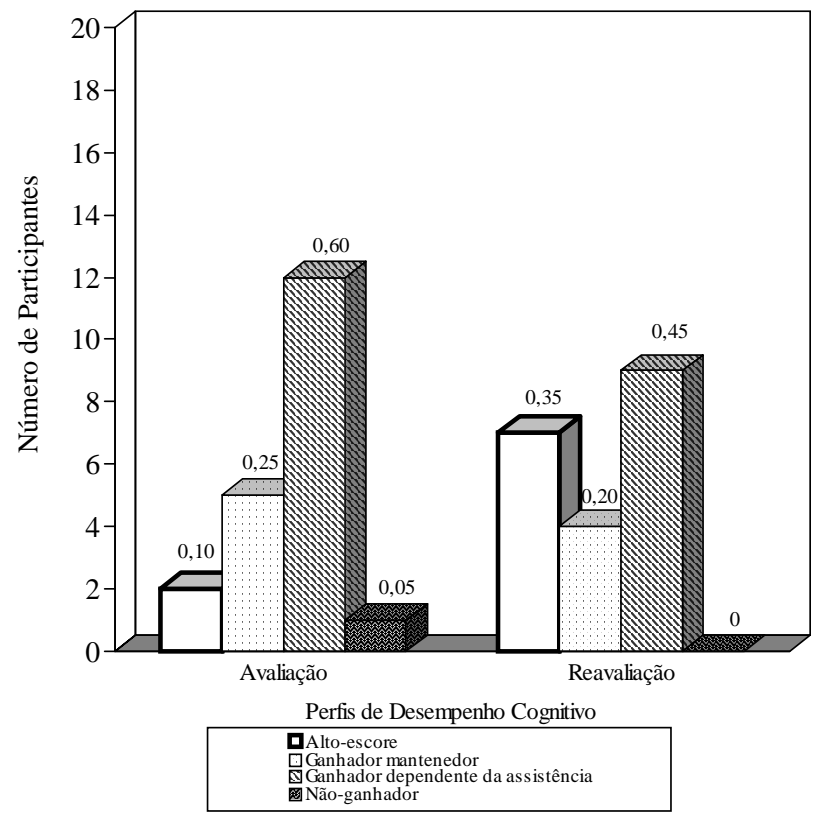

Figura 1. Perfis de desempenho cognitivo obtidos através do Jogo de Perguntas de Busca com Figuras Diversas, na Avaliação e na Reavaliação

apresentando estratégias de formulação de perguntas relevantes de busca de informação, enquanto que 0,60 das crianças alcançaram o perfil ganhador dependente da assistência, ou seja, melhoraram seu desempenho com assistência presente, mas não mantiveram essa melhora 
após a suspensão da ajuda na fase MAN. Duas crianças apresentaram o perfil alto-escore, tendo bom desempenho logo na fase SAJ, e apenas uma criança apresentou perfil de não ganbador, isto é, não houve melhora no desempenho, apesar da ajuda da examinadora.

Comparados através do teste de Wilcoxon, os perfis de desempenho cognitivo na avaliação e na reavaliação apresentaram uma diferença significativa $(p \leq 0,05)$. $\mathrm{Na}$ reavaliação, o subgrupo de perfil cognitivo alto-escore aumentou, representando 0,35 das crianças que conseguiram um bom desempenho logo na fase SAJ, independente da ajuda da examinadora. Conseqüentemente, não houve crianças com perfil de desempenho cognitivo de não ganhador e o subgrupo ganhador diminuiu, especialmente o ganhador dependente da assistência.

Quando se comparam os perfis de desempenho cognitivo tendo como referência o bom desempenho na tarefa, observa-se que na avaliação, a proporção das crianças que apresentaram bom desempenho, mantendoo após ter cessado a ajuda da examinadora, foi em torno de 0,35 (0,10 das crianças com perfil alto-escore e 0,25 com perfil ganbador mantenedor). $\mathrm{Na}$ reavaliação essa proporção aumentou significativamente, representando cerca da metade das crianças $(0,55)$.

Em relação aos resultados obtidos na avaliação psicométrica através do Raven, constatou-se uma variação quanto à classificação do nível intelectual das crianças. Uma proporção maior de crianças $(0,60)$ obteve classificação intelectual definidamente abaixo da média (percentil 10 ou 25), enquanto que 0,25 das crianças obtiveram classificação intelectual definidamente acima da média (percentil 75) e 0,15 classificação de intelectualmente média (percentil 50).

A Tabela 3 apresenta os índices de correlação de postos de Spearman entre os perfis de desempenho cognitivo, na avaliação e na reavaliação, respectivamente, e as variáveis percentil no Raven e idade cronológica das crianças.

De acordo com a Tabela 3, verifica-se que os perfis de desempenho cognitivo alcançados na avaliação assistida, tanto na avaliaģão quanto na reavaliação, foram independentes dos valores de percentil no teste psicométrico de Raven. Porém, houve correlação significativa positiva entre a idade das crianças e o perfil de desempenho cognitivo na avaliação, ou seja, as crianças mais velhas desempenharam-se melhor do que as crianças mais jovens. Essa correlação não se repetiu na reavaliação, podendo sugerir que, após a intervenção psicopedagógica, as crianças mais jovens conseguiram melhorar o nível de seu desempenho, diminuindo a diferença em relação ao desempenho apresentado pelas crianças mais velhas.

\section{Discussão}

A grande variação nos percentis obtidos no Raven confirmou a observação realizada anteriormente por Marturano e colaboradores (1997), de que crianças com queixa de dificuldade de aprendizagem, encaminhadas para atendimento psicológico na área da Saúde, apresentam diversidade quanto ao desempenho intelectual, medido por avaliação psicométrica. No presente trabalho, a classificação intelectual das crianças variou de deficiente até inteligência acima da média.

Por outro lado, mesmo tendo 60\% das crianças apresentado classificação definidamente abaixo da média intelectual no Raven, pode ser observado indicadores de recursos cognitivos nesse grupo. $\mathrm{Na}$ avaliação cognitiva assistida, a maioria das crianças foi capaz de melhorar seu desempenho mediante à assistência da examinadora, independentemente do nível intelectual alcançado na avaliação psicométrica.

Ao receber um suporte de ajuda adequado às suas necessidades, as crianças com problemas de aprendizagem foram capazes de superar a dificuldade em diferenciar aspectos relevantes dos irrelevantes, elevando o nível do seu desempenho durante a solução de um dado problema, conforme encontrado nos estudos de Barton (1988), Brown e Campione (1986) e de Swanson (1995).

Tabela 3. Índices de Correlação de Postos de Spearman (rs) entre os Perfis de Desempenho Cognitivo no PBFD, na Avaliação e na Reavaliação, e o Percentil no Raven e Idade das Crianças

\begin{tabular}{lcc}
\hline & \multicolumn{2}{c}{ Perfil de Desempenho Cognitivo } \\
\hline Avaliação & Reavaliação \\
\hline Raven & 0,39 & 0,30 \\
Idade & $0,49 *$ & 0,35 \\
\hline$* p \leq 0,05$ & &
\end{tabular}


A análise dos dados permitiu verificar diferenças significativas intragrupo em relação ao desempenho cognitivo das crianças na avaliação assistida em situação de resolução de problemas de perguntas de busca de informação e restrição de alternativas. Embora uma proporção considerável das crianças tenha necessitado de ajuda e melhorado com a assistência durante a avaliação, a possibilidade de se beneficiar da assistência e reorganizar o padrão de funcionamento cognitivo na resolução da tarefa variou para cada criança, formando subgrupos diferenciados quanto a indicadores de eficiência e manutenção da aprendizagem, tanto no momento da avaliação quanto da reavaliação.

Os indicadores de desempenho cognitivo analisados nos dois momentos da avaliação assistida revelaram subgrupos com diferentes perfis de desempenho cognitivo. Durante a avaliação, apesar da ajuda da examinadora, houve predomínio de crianças com perfil de desempenho cognitivo ganhador dependente da assistência, que melhoraram o desempenho mas não mantiveram a melhora na fase de manutenção, dependendo da ajuda da examinadora para obter sucesso na resolução de problemas. As crianças que conseguiram manter o bom desempenho após a suspensão da ajuda da examinadora representam um grupo significativamente menor (alto-escore e ganhador mantenedor), e apenas uma criança obteve o perfil de não ganhador, indicando uma dificuldade maior frente à tarefa proposta.

Por outro lado, na reavaliação, cerca da metade das crianças $(0,55)$ conseguiu manter ou melhorar o bom desempenho na resolução da tarefa (alto-escore e ganhador mantenedor), após a suspensão da ajuda da examinadora. $\mathrm{O}$ aumento na proporção de crianças com perfil alto-escore e a ausência daquelas com perfil de nãoganhador sugere que, após receber assistência mais intensiva e prolongada, representada pela intervenção psicopedagógica de curta duração, um número maior de crianças foi capaz de apresentar estratégias de busca de informação mais eficientes. Embora o grupo de crianças com perfil ganhador dependente da assistência tenha diminuído, este continuou representando uma parcela perto da metade das crianças $(0,45)$. Para estas a ajuda oferecida parece não ter sido suficiente para melhorar seu desempenho, havendo necessidade de monitoramento continuado.

Essa variação no desempenho pode ser entendida ao considerar-se que o grupo classificado com queixa de dificuldade de aprendizagem mostrou ser heterogêneo, incluindo crianças com dificuldades ou recursos em níveis diferentes. Além disso, contribuem para essa variação intragrupo, a sensibilidade de cada criança à instrução, ou seja, o potencial individual da criança para mudança e o quanto de ajuda é necessária para que essa mudança ocorra. Com o mesmo nível de ajuda, uma criança pode experimentar melhora significativa no seu desempenho, enquanto outra pode não conseguir os mesmos resultados, conforme já foi observado por outros autores (Brown \& Ferrara, 1985; Linhares, Santa Maria, Escolano \& Gera, 1998; Santa Maria \& Linhares, 1999; Swanson, 1995).

A melhora de desempenho na avaliação assistida, no momento da avaliação, foi mais evidente nas crianças de faixa etária maior, uma vez que as mais jovens apresentaram maior dificuldade na solução da tarefa, necessitando de mais ajuda da examinadora para resolvêla. $\mathrm{Na}$ reavaliação, no entanto, após a dessensibilização da criança para enfrentar situações de aprendizagem, promovida através de um período de intervenção psicopedagógica de curta duração, a relação entre faixa etária e melhora no desempenho não ficou evidenciada.

A relação entre idade e desempenho em tarefas que envolvem a formulação de perguntas para a resolução de problemas, tem sido investigada em estudos anteriores. Segundo pesquisa realizada por Mosher e Hornsby (1966, citado por Barton, 1988) com crianças normais, a idade estaria relacionada ao desempenho em tarefas que exigem formulação de perguntas de busca com exclusão de alternativas. Os autores observaram que, enquanto as crianças de oito anos de idade mostravam pouca habilidade em elaborar perguntas de busca e restrição de alternativas, aquelas com onze anos já apresentavam estratégias eficientes na formulação de perguntas relevantes de busca. Para Courage (1989), essas habilidades desenvolvem-se no decorrer dos primeiros anos escolares, mas a elaboração de perguntas relevantes de busca de informação com restrição de alternativas pode ocorrer mais cedo se a criança for treinada.

Por outro lado, Escolano e Linhares (2000) estudaram aspectos do funcionamento cognitivo de crianças de sete anos da primeira série do ensino fundamental através do Jogo de Perguntas de Busca com Figuras Geométricas e verificaram que, embora jovens, a maioria das crianças da amostra apresentou bom desempenho na tarefa, precisando de pouca ajuda para implementar estratégias eficientes de perguntas relevantes de busca e restrição de alternativas e resolver corretamente os problemas apresentados. Gera e Linhares (1998, citado por Linhares e cols., 1998), por sua vez, ao avaliarem crianças de sete a dez anos de idade, com e sem dificuldade de aprendizagem, através do Jogo de Perguntas de Busca com Figuras Diversas em situação de avaliação assistida, verificaram que o desempenho das crianças na tarefa 
diferiu mais em função da presença de problemas de aprendizagem do que da idade.

Em relação à amostra do presente estudo, observouse que as crianças mais velhas com queixa de dificuldade de aprendizagem mostraram ser mais sensíveis ao suporte instrucional temporário oferecido pela examinadora durante o momento da avaliação, adotando prontamente estratégias eficientes para formular perguntas de busca visando a exclusão de alternativas e resolução correta do problema. As crianças mais jovens com dificuldade de aprendizagem, por outro lado, apresentaram maior dificuldade diante da tarefa durante a avaliação, apesar da assistência presente da examinadora. Provavelmente, a ajuda restrita à situação de avaliação assistida não foi suficiente para que essas crianças pudessem superar as dificuldades durante a resolução da tarefa e adotassem estratégias de perguntas relevantes. Neste caso, foi necessário um período maior de assistência, representado pela intervenção psicopedagógica de curta duração, para que a melhora no desempenho ocorresse. Posteriormente a esse período, verificou-se a diluição da diferença no desempenho entre crianças mais jovens ou mais velhas, ou seja, houve equiparação do nível de desempenho de crianças de idades diferentes, dentro da faixa estudada.

Concluindo, os achados do presente estudo demonstram que ao neutralizar condições adversas de ensino, criando-se uma mini-situação de ensinoaprendizagem, torna-se possível a diferenciação de crianças que precisam de ajuda intensiva e continuada para melhorar o desempenho estratégico para resolver problema, daquelas que com pouca ajuda são capazes de revelar recursos eficientes, as quais podem ter tido sua capacidade cognitiva subestimada por medida psicométrica. Estas últimas podem ser identificadas como "pseudodeficientes" ou "deficientes mediacionais" (Feuerstein e cols., 1980), ou seja, possuem recursos cognitivos ou potencial de aprendizagem além daquele estimado psicometricamente.

A partir do momento em que recursos cognitivos são identificados e não apenas dificuldades, crianças com problemas de aprendizagem podem ser retiradas de categorias diagnósticas estabelecidas por testes psicométricos. Reconhecer a sensibilidade das crianças à mediação ou assistência do outro, permite que seu processo de aprendizagem possa ser redefinido sob um ponto de vista mais otimista, de acordo com a proposta de "experiência de aprendizagem mediada" formulada por Feuerstein e colaboradores (1980).

\section{Referências}

Angelini, A L., Alves, I. C. B., Custódio, E. M. \& Duarte, W. F. (1987). Matrizes Progressivas Coloridas - Escala Especial - Raven. (Padronização Brasileira). São Paulo: Casa do Psicólogo.

Barton, J. A (1988). Problem-solving strategies in learning disabled and normal boys: Developmental and instructional effects. Journal of Educational Psychology, 80(2), 184-191.

Brown, A L. \& Campione, J. C. (1986). Psychological theory and the study of learning disabilities. American Psychologist, 14, 1059-1068.

Brown, A L. \& Ferrara, R. A (1985). Diagnosing zones of proximal development. Em J. V. Wertsch (Org.), Culture communication and cognition: Vygotskian perspectives (pp. 273-305). Cambridge University Press.

Campione, J. C. (1989). Assisted assessment: A taxonomy of approaches and an outline of strengths and weaknesses. Journal of Learning Disabilities, 22(3), 151-165.

Campione, J. C. \& Brown, A. L. (1990). Guided learning and transfer: Implications for approaches to assessment. Em N. Fredericksen, R. Glasser, A. Lesgold \& M. G. Shafto (Orgs.), Diagnostic monitoring of skill and knowledge acquisition (pp. 141-172). Hillsdale: Lawrence Erlbaum.

Courage, M. L. (1989). Children's inquiry strategies in referential communication in the game of twenty questions. Child Development, 60, 877886.

Escolano, A. M. C. (2000). Avaliação cognitiva assistida em situação de resolução de problema na predição do desempenho escolar de crianças de primeira série do primeiro grau. Dissertação de Mestrado não-publicada, Curso de PósGraduação em Psicologia, Universidade de São Paulo. Ribeirão Preto, SP.

Escolano, A. M. C. \& Linhares, M. B. M. (2000). Avaliação cognitiva assistida em situação de resolução de problemas na predição do desempenho escolar de crianças de primeira série do primeiro grau. Em Z. M. M. B.Alves, M. Japur, M. A. Santos, F. C. R. Brunhara, E. F. Rasera, C. P. Simon \& D. J. Cremonezi (Orgs.), III Seminário de Pesquisa - Tomo II (pp.55-63). Ribeirão Preto: Programa de Pós-Graduação em Psicologia, Universidade de São Paulo.

Feuerstein, R., Rand, Y., Hoffman, M. \& Miller, R. (1979). Cognitive modifiability in retarded adolescents: Effects of instrumental enrichment. American Journal of Mental Deficiency, 83(6), 539-550.

Feuerstein, R., Rand, Y., Hoffman, M. \& Miller, R. (1980). Instrumental enrichment. An intervention program for cognitive modifiability. Illinois: Scott, Foresman.

Gera, A. \& Linhares, M. B. M. (1998). Estratégias de perguntas de busca de informação na resolução de problemas de crianças com e sem queixa de dificuldade de aprendizagem [Resumo]. Em Sociedade Brasileira de Psicologia (Org.), Resumos de comunicações científicas, XVIII Reunião Anual de Psicologia (p. 126). Ribeirão Preto: SBP.

Graminha, S. S. V. \& Martins, M. A. O. (1997). Condições adversas na vida de crianças com atraso no desenvolvimento. Medicina, 30, 259 - 267.

Linhares, M. B. M. (1996). Avaliação assistida em crianças com queixa de dificuldade de aprendizagem. Temas de Psicologia, 1, 17- 32.

Linhares, M. B. M. (1998a). Avaliação psicológica de aspectos cognitivos em crianças com queixa de dificuldade de aprendizagem. Em C. A Funayama (Org.), Problemas de aprendizagem: Enfoque multidisciplinar (pp. 41-59). Ribeirão Preto: Legis Summa.

Linhares, M. B. M. (1998b). Atendimento psicopedagógico de crianças em serviço especializado de psicologia infantil na área da Saúde: Uma perspectiva desenvolvimentista. Psicopedagogia, 17(46), 30 - 36.

Linhares, M. B. M. (1998c). Avaliação assistida de crianças com queixa de dificuldade de aprendizagem: Indicadores de eficiência e transferência de aprendizagem em situação de resolução de problemas. Em A. W. Zuardi, E. M. Marturano, M. A. C. Figueiredo \& S. R. Loureiro (Orgs.), Estudos em Saúde Mental (pp.121-147). Ribeirão Preto: Comissão de Pós-graduação em Saúde Mental/FMRP/USP.

Linhares, M. B. M., Parreira, V. L. C., Maturano, A C. \& Sant'Anna, S. C. (1993). Caracterização dos motivos da procura de atendimento infantil em um serviço de psicopedagogia clínica. Medicina, 26(2), 148-160. 
Linhares, M. B. M., Santa Maria, M. R., Escolano, A C. M. \& Gera, A. A. (1998). Avaliação cognitiva assistida: Uma abordagem promissora na avaliação cognitiva de crianças. Temas em Psicologia, 7(3), 231-254.

Lopez, M. A (1983). Características da clientela de clínicas-escola de psicologia em São Paulo. Arquivos Brasileiros de Psicologia, 35(1), 78-92.

Lunt, J. (1994). A prática da avaliação. Em H. Daniels (Orgs.), Vygotsky em foco: Pressupostos e desdobramentos (pp. 219-252). Campinas: Papirus.

Marturano, E. M., Loureiro, S. R., Linhares, M. B. M. \& Machado, V. L. S. (1997). A avaliação psicológica pode fornecer indicadores de problemas associados a dificuldades escolares? Em A W. Zuardi, E. M. Marturano \& S. R. Loureiro (Orgs.), Estudos em Saúde Mental (pp. 11-48). Ribeirão Preto: Comissão de Pós-Graduação em Saúde Mental da Faculdade de Medicina de Ribeirão Preto/USP.

Santa Maria, M. R. (1999). Avaliação cognitiva assistida de crianças com indicação de dificuldade de aprendizagem escolar e deficiência mental. Dissertação de Mestrado não-publicada, Curso de Pós-Graduação em Saúde Mental, Universidade de São Paulo. Ribeirão Preto, SP.

Santa Maria, M. R. \& Linhares, M. B. M. (1999). Avaliação cognitiva assistida de crianças com indicação de dificuldade de aprendizagem escolar e deficiência mental. Psicologia: Reflexão e Crítica, 12, 5-27.
Santos, M. A (1990). Caracterização da clientela de uma clínica psicológica da prefeitura de São Paulo. Arquivos Brasileiros de Psicologia, 42, 70-94.

Swanson, H. L. (1995). Effects of dynamic testing on the classification of learning disabilities: The predictive and discriminant validity of the Swanson-Cognitive Processing Test (S- CPT). Journal of Psychoeducational Assessment, 13, 204-229.

Tzuriel, D. \& Klein, P. S. (1987). Assessing the young child: Children's analogical thinking modifiability. Em C. S. Lidz (Org.), Dynamic assessment: An interactional approach to evaluating learning potencial (pp. 268287). London: Guilford.

Vygotsky, L. S. (1988). A formação social da mente: Desenvolvimento dos processos psicológicos superiores. São Paulo: Martins Fontes. (Original publicado em 1978)

Sobre as autoras:

Silvia Helena Tortul Ferriolli - Psicóloga, mestre em Psicologia no Departamento de Psicologia e Educação da Faculdade de Filosofia, Ciências e Letras de Ribeirão Preto, Universidade de São Paulo e bolsista da CAPES.

Maria Beatriz Martins Linhares - Psicóloga, Especialista em Psicologia Clínica Infantil, Mestre em Educação Especial, Doutora em Ciências (Psicologia Experimental), Professora Doutora do Departamento de Neurologia, Psiquiatria e Psicologia Médica da Faculdade de Medicina de Ribeirão Preto, Universidade de São Paulo, Orientadora nos Cursos de Pós-Graduação em Ciências Médicas - Saúde Mental (FMRP/USP) e Psicologia (FFCLRP/USP) e Pesquisadora do CNPq.

Sonia Regina Loureiro - Psicóloga e Professora Doutora do Departamento de Neurologia, Psiquiatria e Psicologia Médica da Faculdade de Medicina de Ribeirão Preto, Universidade de São Paulo, Orientadora nos Cursos de Pós-Graduação em Ciências Médicas - Saúde Mental (FMRP/ USP) e Psicologia (FFCLRP/USP) e Pesquisadora do CNPq, Coordenadora do Serviço de Psicodiagnóstico (Psiquiatria/HCRP), onde mantém atividades de formação de recursos humanos e de pesquisa com instrumentos e procedimentos de avaliação.

Edna Maria Marturano - Psicóloga e Professora Titular do Departamento de Neurologia, Psiquiatria e Psicologia Médica da Faculdade de Medicina de Ribeirão Preto, Universidade de São Paulo e Pesquisadora do CNPq. 